\title{
Blackcurrant reversion virus: Validation of an improved diagnostic test, accelerating testing in breeding and certification of blackcurrants
}

\author{
A. Dolan ${ }^{\mathrm{a}, *}$, S.A. MacFarlane ${ }^{\mathrm{a}}$, W.J. McGavin ${ }^{\mathrm{a}}$, R.M. Brennan ${ }^{\mathrm{b}}$ and J.W. McNicol ${ }^{\mathrm{c}}$ \\ a Plant Pathology Programme, The James Hutton Institute, Invergowrie, Dundee, UK \\ ${ }^{\mathrm{b}}$ Genetics Programme, The James Hutton Institute, Invergowrie, Dundee, UK \\ ${ }^{\mathrm{c} B i o m a t h e m a t i c s}$ and Statistics Scotland (BioSS), The James Hutton Institute, Invergowrie, Dundee, UK
}

Received 28 February 2011; accepted 26 April 2011

\begin{abstract}
A study was commenced in 2005 to provide a validated diagnostic test for the detection of Blackcurrant reversion virus (BRV) to be used as an alternative to the conventional test recommended for use in the UK certification scheme. A range of cultivars previously virus indexed and held as nuclear stock (Baldwin, Ben Lomond and Ben Tirran) were grafted with known BRV-positive scions and assessed over the following four years. Data was collected through visual observations and buds sampled and tested with a single round RT-PCR using two new primer sets. For the method to be accepted for use in Scotland the detection rate must be equal or better than that of the existing grafting method. Statistical analysis of our results for the detection of both the European (E) and Russian (R) forms of Reversion disease in the three cultivars provides a validation for this test. We suggest that this method provides a faster throughput test for BRV even when no symptoms are visible on the plants, and so is suitable for adoption into the guidelines followed by both UK and European authorities for detection of BRV in blackcurrant propagation material.
\end{abstract}

Keywords: Blackcurrant reversion virus (BRV), RT-PCR, UK certification scheme, EPPO, PHPS, nuclear stock

\section{Introduction}

Reversion is one of the most serious diseases of blackcurrant (Ribes nigrum L.), and is caused by a mite-transmitted virus (Blackcurrant reversion virus, BRV). The disease occurs in most areas where Ribes plants are found, except North America and Australia, and exists in two forms; the European form (E) and the Russian form (R), which have been recognised based on the severity of the symptoms displayed, with the $\mathrm{R}$ form being markedly more severe than the E form. Symptoms common to both forms of the disease are changes in leaf shape, a decrease in hair numbers and an increase in colour intensity on the flower buds [1-4]. Further malformation of the flowers is associated with the $\mathrm{R}$ form, resulting in a proliferation of the sepals (a doubling of the usual five to ten) and a more pronounced colour change of the buds. In the most affected plants, the disease causes sterility with a consequent complete loss of the crop, making BRV a serious impediment to profitable blackcurrant production. Durable resistance to BRV from the wild species $R$. dikuscha has been accessed by blackcurrant breeders, leading to the release of the cultivars 'Golubka' from Russia and 'Ben Gairn' (a 'Golubka' derivative) from Scotland.

${ }^{*}$ Corresponding author: Alison Dolan, Plant Pathology Programme, The James Hutton Institute, Invergowrie, Dundee, DD2 5DA, UK. Tel.: +44 (0) 844928 5428; Fax: +44 (0) 844928 5429; E-mail: Alison.dolan@ hutton.ac.uk. 
The James Hutton Institute is the sole source of Rubus and Ribes nuclear stock material entering the UK soft fruit Certification Scheme and is the centre for UK blackcurrant breeding.

Plants that are maintained as part of the nuclear stock collection following cultivar release, as well as those entering the breeding programme, are tested to ensure that they are free from a range of pathogens, including BRV, following guidelines issued by both UK and European authorities. In Scotland these guidelines are determined by the Scottish Government, in England and Wales by the Department for Environment, Food and Rural Affairs (Defra) and in Europe by the European and Mediterranean Plant Protection Organisation (EPPO). The current recommended test for detection of BRV in blackcurrants is a visual examination over a two year period of recipient plants (of a symptom-expressing cultivar such as Baldwin) that have been grafted with a scion taken from the mother plant that is under test [1]. This assessment regime is costly, labour intensive, time consuming and requires the involvement of staff who are experienced in visual assessment of the disease symptoms.

A reverse transcriptase polymerase chain reaction (RT-PCR) test for BRV, based on the sequence of an isolate of the virus obtained from blackcurrant plants in Finland showing the $\mathrm{R}$ form of reversion disease, has been available since the initial characterization of the virus in 1997 [5]. This method uses capture of the virus onto anti-BRV antibody-coated tubes, followed by reverse transcription and a single-round PCR amplification using primers located in the 3' untranslated region that is shared by both RNA1 and RNA2 of the virus. A variation of this method uses a commercial kit to isolate RNA from leaves of blackcurrant prior to the RT-PCR steps of the detection process [6].

An improvement to the original method was reported [4], in which virus particles were adsorbed to the surface of an enzyme-linked immunosorbent assay (ELISA) plate without prior coating of the plate using anti-BRV antiserum. The trapped virus particles were then lysed by detergent treatment (TritonX-100), and the virus RNA was amplified by RT-PCR with two, different primer pairs, designed using the BRV coat protein sequence. However its evaluation was limited to a small number of plants (eight) and was not statistically analysed; also the PCR amplification was nested, thus increasing the risk of contamination between steps, and was more costly and time consuming than a single round assay. Finally the test was assessed on plants that had been mechanically inoculated with partially purified preparations of the virus and not on plants that were grafted in the conventional manner.

Because of the speed of the technique RT-PCR has been suggested as a useful alternative to the biological (graft) indexing method for BRV detection in blackcurrant propagation stock [3]. However, BRV is known to accumulate at low levels and with an erratic distribution in infected plants, with some stems remaining apparently virus-free or with only the occasional infected bud being found on an otherwise apparently virus-free stem [7]. It is important therefore that the sample taken is from across the whole plant and in sufficient numbers, five buds, to detect the virus (3).

Thus far there have been no reports of any systematic, statistically robust study of the efficacy of RT-PCR detection of BRV. This work looks at two new single- round PCR methods and validates them against the conventional grafting method. This is done in a manner necessary to meet the stringent requirements for the recognition of a proposed alternative test by the UK and European plant health regulatory bodies. Scions from the collection of known BRVpositive and -negative plants held at The James Hutton Institute were grafted onto three blackcurrant cultivars and, in parallel, examined both visually and using RT-PCR to rigorously evaluate the new tests in comparison to the conventional test in detecting the virus.

\section{Materials and methods}

\subsection{Experiment design}

In February 2005 thirty hardwood cuttings were taken from virus indexed (BRV-negative) plants of each of three blackcurrant cultivars growing in the high health facility at The James Hutton Institute. The chosen cultivars Ben Lomond, Ben Tirran and Baldwin are all known to be susceptible to the gall mite and the reversion agent [1] [8] and Baldwin is also the indicator plant recommended for the detection of BRV by the UK and European authorities.

The cuttings were propagated in an aphid-proof glasshouse and divided into groups to receive different treatments. Each plant was grafted with two scions, except for five plants of each cultivar which had no graft. Ten plants from each of the three cultivars were grafted with scions taken from (1) plants carrying the European (E) form of reversion, or (2) plants carrying the Russian (R) form of reversion. In addition, five plants were grafted with scions taken from virus-free plants. 
The mother plants, which were used to provide the BRV-infected scions, were accessed from a collection of previously virus-indexed BRV-infected cultivars maintained at The James Hutton Institute. Those infected with the $\mathrm{R}$ form of the disease were accessed from a separate, purpose built glasshouse which contains plants infected with non-indigenous pathogens.

The grafted (recipient) plants were visually assessed for BRV-induced leaf and flower bud symptoms in 2006; from 2007 to 2009 the plants were assessed visually and also tested by RT-PCR using two different BRV-specific primer sets. It was decided to assess dormant buds and not young leaves to assist in spreading the workload of tests required more evenly throughout the year.

\subsection{RNA extraction method}

After fruiting and subsequent leaf fall, blackcurrant plants enter a dormant state over the winter (from about November to March in Scotland). Five dormant (leaf) buds were collected randomly from each plant (in an attempt to overcome any variations in virus distribution within each plant) and processed as a group. Buds were taken only from the recipient plants and not from the grafted scions, thus ensuring any BRV detection was of transmitted virus and not residual, source virus.

The buds were then stored at $4^{\circ} \mathrm{C}$, if to be tested within seven days, or at $-80^{\circ} \mathrm{C}$, if to be tested later using an revised RNA extraction method (with small changes to volume of supernatant used, times and speeds of centrifugation) of that described previously (3).

For each plant, the five buds were homogenised using a pestle and mortar with $2 \mathrm{ml}$ of phosphate-buffered saline (PBS) containing $0.05 \%$ Tween-20, $2 \%$ polyvinylpyrollidone (PVP) and $0.2 \%$ ovalbumin (PBS-TPO).

The extract was centrifuged for $1 \mathrm{~min}$ at $14000 \mathrm{rpm}$ and $100 \mu \mathrm{l}$ of the supernatant was transferred to a well of an ELISA plate (Nunc) and incubated at $4{ }^{\circ} \mathrm{C}$ overnight. The plate was emptied and washed once in PBS-TPO and then again in water before being placed immediately on ice. To each well $20 \mu \mathrm{l}$ of $3 \%$ TritonX-100 solution was added, and the virus particles were disrupted by drawing the fluid up into a $10 \mu$ l pipette tip and expelling it back into the well three times. An aliquot of the lysed virus suspension was immediately examined by RT-PCR.

\subsection{RT-PCR amplification}

To design new primers pairs for BRV detection, the sequences of BRV RNA1 (GenBank accession number AF368272) and BRV RNA2 (GenBank accession number AF020051) were analysed using the programme Primer3, to produce a selection of seventeen primer pairs distributed across the BRV genome. Two pairs, BRV1-8F (5'GAATGTGGTGGTGCAGTGTC3')/BRV1-8 R (5'CCCAACAAAAACTGGATGCT3') (amplifying a 247nt region from nucleotides 2517 to 2763 in BRV RNA1) and BRV1-10F (5'AGGCTTCTCGCACAACATCT3')/BRV1$10 \mathrm{R}$ (5' CAAGGGAGGGTCGAGTTACA3') (amplifying a 327nt region from nucleotides 2021 to 2347 in BRV RNA1) produced the strongest amplification of BRV sequences (data not shown) and were used during the validation experiments, both without the need to do a nested reaction.

Tubes containing an RT-PCR bead (Ready-To-Go RT-PCR beads, GE Healthcare) were placed on ice, to which was added $38 \mu \mathrm{l}$ of sterile water, $1 \mu \mathrm{l}$ of each primer $(20 \mathrm{pmol} / \mu \mathrm{l})$ and $10 \mu \mathrm{l}$ of virus lysate, from the ELISA plate.

For primer set BRV1-8F/8R the RT-PCR conditions were $42^{\circ} \mathrm{C}$ for $60 \mathrm{~min}$., $94^{\circ} \mathrm{C}$ for $5 \mathrm{~min}$., and then 40 cycles of $94^{\circ} \mathrm{C}$ for $1 \mathrm{~min} ., 57^{\circ} \mathrm{C}$ for $1 \mathrm{~min}$., $72^{\circ} \mathrm{C}$ for $1 \mathrm{~min}$., followed by $72^{\circ} \mathrm{C}$ for $10 \mathrm{~min}$. For primer set BRV1-10F/10 R the RT-PCR conditions were $42^{\circ} \mathrm{C}$ for $60 \mathrm{~min} ., 4^{\circ} \mathrm{C}$ for $5 \mathrm{~min}$., and then 40 cycles of $94^{\circ} \mathrm{C}$ for $1 \mathrm{~min}$., $60^{\circ} \mathrm{C}$ for $1 \mathrm{~min}$., $72^{\circ} \mathrm{C}$ for $1 \mathrm{~min}$., followed by $72^{\circ} \mathrm{C}$ for $10 \mathrm{~min}$. RT-PCR products were resolved by electrophoresis through a $1.5 \%$ agarose gel, and visualised by ethidium bromide staining and UV illumination.

\subsection{Statistical methods}

Data analysed were the number of positive plants according to visual inspection and PCR amplification in each group of 5, from each of the six treatment combinations ( 3 mother plant cultivars by 2 virus types [E and R]). Detection rates were estimated using a generalised linear model with binomial error and logit link, fitting effects for mother cultivar, virus type and their interaction. All analyses used Genstat for Windows, $13^{\text {th }}$ edition (VSN International Ltd, Hemel Hempstead, UK). 


\section{Results}

The experiments described in this work were started in March 2005 with grafting of scions from healthy or infected mother plants onto disease-free recipient plants of three cultivars, Baldwin, Ben Lomond and Ben Tirran. Non-grafted plants of these cultivars were also propagated, as an additional negative control for the experiments.

All healthy and infected mother plants were only visually assessed for BRV before their scions were used for grafting. However after PCR testing with both primer pairs not all infected mother plants tested positive for BRV. This was especially true for those plants previously thought to be infected with the $\mathrm{E}$ form of reversion. These findings account for the unequal plant numbers reported below as only the results from the infected mothers, which tested positive by both visual observation and PCR, were included in the analysis. No reversion symptoms were observed in any plant in the experiment in 2006 and so no RT-PCR analysis was done during that year. All negative control plants continued to test negative throughout 2007-2009 both when assessed visually and by PCR amplification. The results of the experiments are presented below and in Tables 1a (2007), 1b (2008) and 1c (2009).

\subsection{7}

In 2007, bud symptoms (as described above) were seen in 2 of 6 Baldwin and 1 of 7 Ben Lomond plants grafted with the $\mathrm{E}$ form of reversion, whereas for the $\mathrm{R}$ form symptoms were apparent in more plants (10 of 10 Baldwin and 9 of 10 Ben Lomond). In contrast, none of the Ben Tirran plants showed symptoms of reversion with either of the forms. Baldwin was the only cultivar to show leaf symptoms, 2 of 6 and 10 of 10 for both the E form and R form, respectively. Ben Tirran showed no leaf symptoms and Ben Lomond only 1 of 10 for the R form.

Using primer set BRV1-8F/8 R the E form of reversion was detected in 1 of 6 Baldwin plants, 2 of 7 Ben Lomond plants but none of the 6 Ben Tirran plants. The R form of reversion was detected in 8 of 10 Baldwin plants, 10 of 10 Ben Lomond plants, and 6 of 9 Ben Tirran plants.

Using primer set BRV1-10F/10 R the E form of reversion was detected in 1 of 5 Baldwin plants, 4 of 7 Ben Lomond plants, and 1 of 6 Ben Tirran plants. The R form of reversion was detected in 9 of 9 Baldwin plants, 10 of 10 Ben Lomond plants, and 7 of 9 Ben Tirran plants.

\subsection{8}

In 2008, bud symptoms were seen in Baldwin (E form 2 of 6 plants; $R$ form 8 of 9 plants), Ben Lomond (E form 3 of 6 plants; R form 9 of 10 plants) and Ben Tirran (E form 1 of 5 plants; R form 5 of 7 plants). No plants in any of the three cultivars were found to display flat leaf symptoms associated with the $\mathrm{E}$ form of reversion, and for the R form only Ben Lomond (1 of 10) and Ben Tirran (4 of 9) showed these characteristics.

Using primer set BRV1-8F/8 R the $\mathrm{E}$ form of reversion was detected in 1 of 6 Baldwin plants, 3 of 7 Ben Lomond plants but none of the 5 Ben Tirran plants. The R form of reversion was detected in 7 of 9 Baldwin plants, 9 of 9 Ben Lomond plants, and 7 of 8 Ben Tirran plants.

Using primer set BRV1-10F/10 R the E form of reversion was detected in 1 of 6 Baldwin plants, 3 of 7 Ben Lomond plants, and 3 of 5 Ben Tirran plants. The R form of reversion was detected in 7 of 9 Baldwin plants, 10 of 10 Ben Lomond plants, and 7 of 8 Ben Tirran plants.

\subsection{9}

In 2009, reversion symptoms were seen in Baldwin (E form 2 of 6 plants; R form 9 of 9 plants), Ben Lomond ( $\mathrm{E}$ form 3 of 7 plants; $\mathrm{R}$ form 10 of 10 plants) and Ben Tirran (R form 5 of 8 plants; no symptoms seen with $\mathrm{E}$ form). No leaf symptoms were detected for either form in all three cultivars.

Using primer set BRV1-8F/8R the $\mathrm{E}$ form of reversion was detected in 2 of 6 Baldwin plants, 3 of 7 Ben Lomond plants and 1 of 5 Ben Tirran plants. The R form of reversion was detected in 8 of 9 Baldwin plants, 10 of 10 Ben Lomond plants, and 6 of 8 Ben Tirran plants. 
Using primer set BRV1-10F/10R the E form of reversion was detected in 1 of 6 Baldwin plants, 4 of 7 Ben Lomond plants, and 3 of 5 Ben Tirran plants. The R form of reversion was detected in 8 of 9 Baldwin plants, 10 of 10 Ben Lomond plants, and 7 of 8 Ben Tirran plants.

\section{Discussion}

The aim of this study was to develop and validate a robust RT-PCR diagnostic test which could detect BRV as well as or better than the conventional grafting test used at present in the UK certification scheme. It was also our intention to provide measures of the precision of estimated detection rates. An optimised RT-PCR test that detects $\mathrm{R}$ and $\mathrm{E}$ forms of the virus equally well would greatly speed up the testing process, perhaps requiring only two to three days rather than the two years needed for grafting, and thereby reduce testing costs. Screening of material in breeding programmes would also be greatly simplified, allowing material to be moved rapidly out of nuclear stock facilities to propagators.

It has previously been noted that the length of time, post BRV-inoculation, necessary for visual symptoms to become apparent differs according to the method of infection (grafting versus mechanical inoculation) and the growth conditions of the plants (field- or glasshouse-grown) but that three to four years may be required for full reversion symptoms to develop [9] [3]. Symptoms of R form disease, being more pronounced, also become apparent more quickly than do E form symptoms.

In our study, after 2 years (2007) bud symptoms of $\mathrm{R}$ form reversion were observed in the majority of Baldwin and Ben Lomond plants but symptoms of E form reversion were seen in fewer plants of these two cultivars. However, no symptoms of either form were observed in Ben Tirran plants.

Nevertheless, the BRV8F/8 R primer set detected the virus at this time in Ben Tirran, although only for the $\mathrm{R}$ form. In contrast, the BRV10F/10 R primer set detected the virus with both forms of BRV in all three cultivars, thus providing very early detection of the disease in the absence of visual symptoms. The formal statistical analyses (Table 1a-c) show that, in general, the RT-PCR diagnostic tests generate detection rates equally as good as those of the visual assessments and that their detection rates are estimated with the same degree of precision (standard error) as the visual tests. However the rapidity of the test makes it superior to the conventional method.

In 2008 a higher detection rate was generally recorded for all methods (visual and RT-PCR), for the presence of the virus in both forms of the disease and in each of the three cultivars. This was probably due to an incremental build up of infection in the plants. The results also revealed that infection has to be established for at least three years before the conventional method of visual assessment can be relied upon to detect the virus in all cultivars. The late detection by this method, compared to the BRV10F/10 R RT-PCR diagnostic method, would prevent early removal of infected plants by a grower. This would increase the risk of spread of the disease from those infected plants to surrounding plants in the plantation, which may ultimately lead to a more severe yield reduction in the crop.

The $\mathrm{E}$ form of the virus in Ben Tirran was recorded, when visually inspected, in 2008 but not in 2009. This may be due to the presence of only a very low level of infection, or the visual symptoms being more difficult to assess for the $\mathrm{E}$ form compared to the $\mathrm{R}$ form.

No leaf symptoms of the E and R form BRV were observed in 2009 and very few in 2007 and 2008. The exception to this was the $100 \%$ detection rate recorded for the $\mathrm{R}$ form in cv. Baldwin in 2007. The corresponding detection rates for 2008 and 2009 were $0 \%$. These findings confirmed that determining the presence of the pathogen by leaf symptoms alone is an unreliable method and should not be used when certifying stock.

BRV was not detected in every plant that had been grafted with virus-infected scions. However, as this was consistent across all detection methods the conclusion drawn was that grafting of scions from a known infected plant onto a recipient host plant does not always guarantee transfer of the infection. A similar observation was reported previously [10]. This result may be due to the erratic distribution of the virus, so that sometimes the scions used may not themselves have been infected. Alternatively, 'inoculating' a plant using this method, even when the scion is infected, may for some species have only a limited success rate. In contrast, $100 \%$ infection was observed when buds from naturally infected field-grown plants (for a period of at least ten years) with the E form of reversion were collected and tested by RT-PCR (data not shown). 
Table 1

Tables 1a (2007), 1b (2008) and 1c (2009). Observed and estimated detection data for visual inspection of disease symptoms and RT-PCR amplification of BRV. Estimated detection rate standard errors are derived from a generalized linear model with logit link and binomial error, fitting Host plant and BRV strain. No evidence of Host plant by BRV strain interaction was detected

\begin{tabular}{|c|c|c|c|c|c|c|c|c|}
\hline \multirow{3}{*}{$\begin{array}{l}\text { Host } \\
\text { Plant }\end{array}$} & \multicolumn{4}{|c|}{ BRV E form } & \multicolumn{4}{|c|}{ BRV R form } \\
\hline & Plants & BRV & Estimated detec- & S.E. & Plants & BRV & Estimated detec- & S.E. \\
\hline & Grafted & Detected & tion rate & & Grafted & Detected & tion rate & \\
\hline
\end{tabular}

\section{Table 1a}

\section{Results}

Bud - visual inspection

\begin{tabular}{|c|c|c|c|c|c|c|c|c|}
\hline $\mathrm{Ba}$ & 6 & 2 & 0.33 & 0.19 & 10 & 10 & 1.00 & 0.00 \\
\hline BL & 7 & 1 & 0.14 & 0.13 & 10 & 9 & 0.90 & 0.09 \\
\hline BT & 5 & 0 & 0.00 & 0.00 & 8 & 0 & 0.00 & 0.00 \\
\hline \multicolumn{9}{|c|}{ Leaf - visual inspection } \\
\hline $\mathrm{Ba}$ & 6 & 2 & 0.33 & 0.19 & 10 & 10 & 1.00 & 0.00 \\
\hline BL & 7 & 0 & 0.00 & 0.00 & 10 & 1 & 0.10 & 0.09 \\
\hline BT & 6 & 0 & 0.00 & 0.00 & 9 & 0 & 0.00 & 0.00 \\
\hline \multicolumn{9}{|c|}{ Bud RT-PCR - primer pair BRV1-8F/8R } \\
\hline $\mathrm{Ba}$ & 6 & 1 & 0.17 & 0.15 & 10 & 8 & 0.80 & 0.13 \\
\hline BL & 7 & 2 & 0.29 & 0.17 & 10 & 10 & 1.00 & 0.00 \\
\hline BT & 6 & 0 & 0.00 & 0.00 & 9 & 6 & 0.67 & 0.16 \\
\hline \multicolumn{9}{|c|}{ Bud RT-PCR - primer pair BRV1-10F/10R } \\
\hline $\mathrm{Ba}$ & 5 & 1 & 0.20 & 0.18 & 9 & 9 & 1.00 & 0.00 \\
\hline BL & 7 & 4 & 0.57 & 0.19 & 10 & 10 & 1.00 & 0.00 \\
\hline BT & 6 & 1 & 0.17 & 0.15 & 9 & 7 & 0.78 & 0.14 \\
\hline
\end{tabular}

Table-1b

\section{Results}

Bud - visual inspection

$\begin{array}{ll}\mathrm{Ba} & 6 \\ \mathrm{BL} & 6 \\ \mathrm{BT} & 5\end{array}$

Leaf - visual inspection

$\begin{array}{ll}\text { Ba } & 6 \\ \text { BL } & 7 \\ \text { BT } & 6\end{array}$

Bud RT-PCR - primer pair BRV1-8F/8R

\begin{tabular}{|c|c|c|c|c|c|c|c|c|}
\hline $\mathrm{Ba}$ & 6 & 1 & 0.17 & 0.15 & 9 & 7 & 0.78 & 0.14 \\
\hline BL & 7 & 3 & 0.43 & 0.19 & 9 & 9 & 1.00 & 0.00 \\
\hline BT & 5 & 0 & 0.00 & 0.00 & 8 & 7 & 0.87 & 0.12 \\
\hline \multicolumn{9}{|c|}{ Bud RT-PCR - primer pair BRV1-10F/10R } \\
\hline $\mathrm{Ba}$ & 6 & 1 & 0.17 & 0.15 & 9 & 7 & 0.78 & 0.14 \\
\hline BL & 7 & 3 & 0.43 & 0.19 & 10 & 10 & 1.00 & 0.00 \\
\hline BT & 5 & 3 & 0.60 & 0.22 & 8 & 7 & 0.87 & 0.12 \\
\hline
\end{tabular}


Table 1

(Continued)

\begin{tabular}{|c|c|c|c|c|c|c|c|c|}
\hline \multirow[b]{2}{*}{$\begin{array}{l}\text { Host } \\
\text { Plant }\end{array}$} & \multicolumn{4}{|c|}{ BRV E form } & \multicolumn{4}{|c|}{ BRV R form } \\
\hline & $\begin{array}{c}\text { Plants } \\
\text { Grafted }\end{array}$ & $\begin{array}{c}\text { BRV } \\
\text { Detected }\end{array}$ & $\begin{array}{c}\text { Estimated detec- } \\
\text { tion rate }\end{array}$ & S.E. & $\begin{array}{c}\text { Plants } \\
\text { Grafted }\end{array}$ & $\begin{array}{c}\text { BRV } \\
\text { Detected }\end{array}$ & $\begin{array}{c}\text { Estimated detec- } \\
\text { tion rate }\end{array}$ & S.E. \\
\hline \multicolumn{9}{|c|}{ Table 1c } \\
\hline \multicolumn{9}{|c|}{2009 Results } \\
\hline \multicolumn{9}{|c|}{ Bud - visual inspection } \\
\hline $\mathrm{Ba}$ & 6 & 2 & 0.33 & 0.19 & 9 & 9 & 1.00 & 0.00 \\
\hline BL & 7 & 3 & 0.43 & 0.19 & 10 & 10 & 1.00 & 0.00 \\
\hline BT & 5 & 0 & 0.00 & 0.00 & 8 & 5 & 0.63 & 0.17 \\
\hline \multicolumn{9}{|c|}{ Leaf - visual inspection } \\
\hline $\mathrm{Ba}$ & 6 & 0 & 0.00 & 0.00 & 10 & 0 & 0.00 & 0.00 \\
\hline BL & 7 & 0 & 0.00 & 0.00 & 10 & 0 & 0.00 & 0.00 \\
\hline BT & 6 & 0 & 0.00 & 0.00 & 9 & 0 & 0.00 & 0.00 \\
\hline \multicolumn{9}{|c|}{ Bud RT-PCR - primer pair BRV1-8F/8R } \\
\hline $\mathrm{Ba}$ & 6 & 2 & 0.33 & 0.19 & 9 & 8 & 0.89 & 0.10 \\
\hline BL & 7 & 3 & 0.43 & 0.19 & 10 & 10 & 1.00 & 0.00 \\
\hline BT & 5 & 1 & 0.20 & 0.18 & 8 & 6 & 0.75 & 0.15 \\
\hline \multicolumn{9}{|c|}{ Bud RT-PCR - primer pair BRV1-10F/10R } \\
\hline $\mathrm{Ba}$ & 6 & 1 & 0.17 & 0.15 & 9 & 8 & 0.89 & 0.10 \\
\hline BL & 7 & 4 & 0.57 & 0.19 & 10 & 10 & 1.00 & 0.00 \\
\hline BT & 5 & 3 & 0.60 & 0.22 & 8 & 7 & 0.88 & 0.12 \\
\hline
\end{tabular}

$\mathrm{Ba}=$ Baldwin, $\mathrm{BL}=$ Ben Lomond and $\mathrm{BT}=$ Ben Tirran.

Two main practical points arise from the results of this study. Firstly, RT-PCR detection is as effective as the currently recommended 2 year visual inspection regime in detecting BRV, and more so when using the BRV10F/10R primer pair for early detection of both E and R forms of BRV in Ben Tirran. Secondly, R-isolates of BRV are detected much more readily and consistently by both RT-PCR amplification and visual assessment than are E-isolates of the virus. This latter finding might be caused by the E-isolate accumulating to lower levels or being less widespread in the plant than the R-isolate. Nevertheless, the detection of both forms of the virus, using the BRV10F/10 R primer set, in a range of different cultivars as early as two years after infection, supports its use as an optimised, reliable, effective and, most importantly, validated PCR test for BRV. This testing method should therefore be considered as an alternative to the existing graft method used within the UK certification scheme and EPPO guidelines.

\section{Acknowledgments}

This work was funded by the Scottish Government. We thank Piritta Lohela, Yvonne Pitkin, James Lynott, Arundhuti Ghatak and Marcin Skiba for technical assistance throughout the project.

Enquiries and further information on the Certification Scheme in Scotland should be directed to: hort.marketing@ scotland.gsi.gov.uk, http://www.fera.defra.gov.uk/plants/plantHealth/phps.cfm, http://www.eppo.org/STANDARDS/ standards.htm.

\section{References}

[1] A.N. Adams and J.M. Thresh, Reversion of black currant, In: Virus Diseases of Small Fruits, R.H. Converse, Ed., USDA Agriculture Handbook No. 631, 1987, pp. 133-136.

[2] A. Lemmetty and K. Lehto, Successful back-inoculation confirms the role of black currant reversion associated virus as the causal agent of reversion disease, European Journal of Plant Pathology 105 (1999), 297-301. 
[3] A.T. Jones, Important virus diseases of ribes, their diagnosis, detection and control, Acta Horticulturae 585 (2002), $279-285$.

[4] A.T. Jones and W.J. McGavin, Improved PCR detection of Blackcurrant reversion virus in Ribes and further evidence that it is the causal agent of reversion disease, Plant Disease 86 (2002), 1333-1338.

[5] A. Lemmetty, S. Latvala, A.T. Jones, P. Susi, W.J. McGavin and K. Lehto, Purification and properties of a new virus from blackcurrant, its affinities with nepoviruses and its close association with blackcurrant reversion disease, Phytopathology 87 (1997), 404-413.

[6] J. Přibylová, J. Špak, K. Petrzik, D. Kubelková and V. Špaková, Sequence comparison and transmission of Blackcurrant reversion virus isolates in black, red and white currants with black currant reversion disease and full blossom disease symptoms, European Journal of Plant Pathology 121 (2008), 67-75.

[7] S. Latvala, P. Susi, A. Lemmetty, S. Cox, A.T. Jones and K. Lehto, Ribes host range and erratic distribution within plants of blackcurrant reversion associated virus provide further evidence for its role as the causal agent of reversion disease, Annals of Applied Biology 131 (1997), 283-295.

[8] A.T. Jones, R.M. Brennan, W.J. McGavin and A. Lemmetty, Galling and reversion disease incidence in a range of blackcurrant genotypes, differing in resistance to the blackcurrant gall mite (Cecidophyopsis ribis) and blackcurrant reversion disease, Annals of Applied Biology 133 (1998), 375-384.

[9] A.T. Jones, Black currant reversion disease - the probable causal agent, eriophyid mite vectors, epidemiology and prospects for control, Virus Research 71 (2000), 71-84.

[10] J. Spak, D. Kubelková, J. Přibylová and V. Špaková, Assessment of the resistance of Ribes cultivars to Blackcurrant reversion virus, Acta Hort 838 (2009), ISHS 2009. 